\title{
Effect of Micro/Nano-Patterned Surfaces on Cell Adhesion of Ca9-22 cells*
}

\author{
Naoyuki Kaga, ${ }^{\dagger}$ Rumi Horiuchi, and Atsuro Yokoyama \\ Department of Oral Functional Prosthodontics, Hokkaido University Graduate School of Dental Medicine, \\ Kita 13 Nishi 7 Kita-ku Sapporo 060-8586, Japan
}

Tsukasa Akasaka and Yasuhiro Yoshida

Department of Biomedical Materials and Engineering,

Hokkaido University Graduate School of Dental Medicine,

Kita 13 Nishi 7 Kita-ku Sapporo 060-8586, Japan

(Received 17 October 2016; Accepted 2 January 2017; Published 11 February 2017)

\begin{abstract}
Surface topography influences cell growth and differentiation. In this study, we used a nano-imprinting method to develop a titanium sheet with $500 \mathrm{~nm}-, 1 \mu \mathrm{m}-$, and $2 \mu \mathrm{m}$-wide grooved and pillared structures. We investigated the effects of the surfaces with the micro- and nano-structures on Ca9-22 cell adhesion and proliferation. Ca9-22 cells were cultured in DMEM containing 10\% fetal bovine serum and counted adhered cells at 1 and $24 \mathrm{~h}$ postculture. Scanning electron microscopy was used to assess cell morphology. Immunofluorescence cell staining was used to evaluate vinculin formation to observe the presence of focal contacts at $24 \mathrm{~h}$. There was no difference in cell adhesion between cells cultured on a plane or groove after $1 \mathrm{~h}$. However, at $24 \mathrm{~h}$, the adhesion of cells cultured on the groove was reduced. In addition, the cell adhesion count on the pillar was less than that of cells cultured on a plane at both 1 hour and $24 \mathrm{~h}$ post-seeding. Furthermore, in the groove of the Ti sheet after 1 and $24 \mathrm{~h}$, cell expansion occurred in the grooved direction. These results demonstrate that the micro and nano-grooved and pillared structures on the titanium sheet control Ca9-22 cell adhesion and orientation. [DOI: 10.1380/ejssnt.2017.1]
\end{abstract}

Keywords: Scanning electron microscopy; Surface structure, morphology, roughness, and topography; Titanium; Ca9-22; Groove; Pillar

\section{INTRODUCTION}

Dental implants are widely used clinically to replace missing teeth in the mandible and/or maxilla. After implant insertion, the implant surface attaches to the gingiva, restricting bacterial invasion. Subsequently, the implant surface maintains osseointegration by direct contact with living bone. However, infection is one of the risk factors for the failure of dental implants [1, 2], and an effective treatment for peri-implantitis has not been established. It is important to apply a micro/nano-patterned structure to the dental implant surface for early osseointegration and prevention of peri-implantitis.

Dental implant bodies penetrate the oral mucosa and may be exposed to infectious agents. Therefore, to prevent inflammation around the implant area, biological blockades with soft tissue adhesion patterns oriented along the direction of and perpendicularly to the dental implant long axis are important. Biological blockades based on soft tissue around the dental implant area play an important role in the success of dental implant treatment. Adhesion to soft tissue toward the implant body and abutment appears to be important for preventing intrusion of the epithelia and bacteria, as well as inflammation around the implant area [3,4]. Specifically, control of the adhesion, expansion, migration, and differentiation in epithelial and fibroblast cells [5-7] is necessary, as biological blockades with soft tissue adhesion patterns are oriented along the implant long axis and perpendicularly. Because grooved structures are attached to the implant

\footnotetext{
* This paper was presented at the 8th Asian Conference on Nanoscience and Nanotechnology (AsiaNANO 2016), Sapporo Convention Center, Sapporo, Japan, October 10-13, 2016.

† Corresponding author: n.kaga@den.hokudai.ac.jp
}

body surface, several studies have explored the suppression of epithelial deep proliferation, arrays in the fiber perpendicular direction, and travel of osteoblast cells to promote morphological formation toward the deep parts of the grooves $[8,9]$. Nurthdurft et al. cultured fibroblast and epithelial cells on zirconium and titanium, and the epithelial cells demonstrated a tendency for cell expansion, which was enhanced on smooth surfaces or machine finish surfaces compared to rough surfaces. Accordingly, the proliferation of cells on smooth and machine finish surfaces was higher than that of cells cultured on zirco- (a)

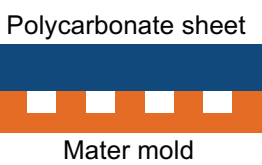

(b)

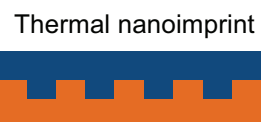

(c)

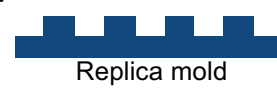

(d)

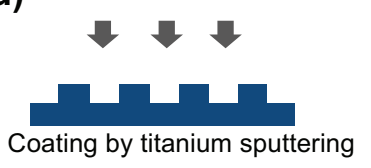

(e)

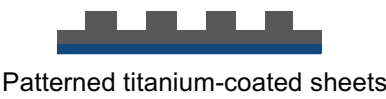

(f)

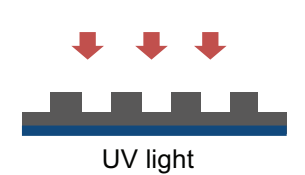

FIG. 1. Schematic representation of the fabrication of micro/nano-patterned titanium-coated sheets. (a) Setting the polycarbonate sheet on micro-/nano-patterned master mold. (b) Pressing on the master mold using a thermal nanoimprint apparatus. (c) Peeling off the replica mold of the patterned polycarbonate sheet. (d) Coating by titanium sputtering on the replica mold. (e) The resulting patterned titanium sheet. (f) Sterilization under UV light. 
nium or titanium [10]. In an in vitro study of epithelial cells on substrates with different surface shapes, cells proliferated faster on smooth surfaces than on rough surfaces [7]. It has also been reported that adhesion of fibroblast cells is improved on mechanically polished or smooth titanium surfaces with grooved structures and other directionality compared to rough surfaces without directionality [11]. Thus epithelial cell adhesion or proliferation is better on smooth than on rough surfaces without directionality. However, the appropriate structures for dental implant body and abutment have not been established. Suppressing epithelial downgrowth is important for preventing peri-implantitis.

Therefore, in this study, we fabricated titanium sheets with micro- and nano-grooved and pillared structures and examined the cell adhesion and focal adhesions of epithelial-like cells (Ca9-22). We also evaluated changes in the cell adhesion and orientation of epithelial cells caused by the micro- and nano-grooved and pillared structures on titanium surfaces.

\section{EXPERIMENTAL}

\section{A. Fabrication of titanium-coated nano-/micro-patterned sheets}

Figure 1 shows the procedure for fabricating micro/nano-grooved and pillared titanium-coated sheets (Tisheets). A quartz master mold was used (Kyodo International Inc., Kawasaki, Japan). Six areas of $5 \times 5 \mathrm{~mm}^{2}$ used in this study were patterned with ridge widths of $500 \mathrm{~nm}, 1 \mu \mathrm{m}$, and $2 \mu \mathrm{m}$; a groove height or depth of $500 \mathrm{~nm}$; widths of $500 \mathrm{~nm}, 1 \mu \mathrm{m}$, and $2 \mu \mathrm{m}$; and a hole depth of $500 \mathrm{~nm}$. A polycarbonate sheet (PC; Sugawara Technology Corp., Tokyo, Japan) was pressed on the master mold at $0.2 \mathrm{MPa}$ and $175^{\circ} \mathrm{C}$ for 4 min using a thermal nanoimprint apparatus (AH-1TC, Shimadzu Corp., Kyoto, Japan), and then was gradually cooled to $23^{\circ} \mathrm{C}$ (Fig. 1(a) and 1(b)). Subsequently, the sheet was carefully peeled off to obtain a replica PC mold (Fig. 1(c)). The replica PC sheet was coated with titanium sputtering (HSR412, Shimadzu Corp.) (Fig. 1(d)). The Tisheet was gently transferred into a culture dish and fixed (Fig. 1(e)). All sheets were sterilized under UV light for 30 min (Fig. 1(f)).

For scanning electron microscope (SEM; S-4000, Hitachi High-Tech Fielding Corp., Tokyo, Japan) observation, the patterned Ti sheets were sputter-coated with PtPd using a sputtering apparatus (E-1030, Hitachi HighTech Fielding Corp.). Next, SEM images of the surface patterns were obtained. Surface topography and roughness of the patterned Ti-sheets were measured with a 3D laser microscope (VK-X200; Keyence Corp., Osaka, Japan). The average surface roughness $\left(\mathrm{R}_{\mathrm{a}}\right)$ was measured on six grooved or pillared areas $\left(10 \times 10 \mu \mathrm{m}^{2} /\right.$ field $)$.

The hydrophilicity of the surface of each sample was investigated using a contact angle meter (DMs-200, Kyowa Electronic Instruments, Tokyo, Japan). Droplets of ultrapure water $(2 \mu \mathrm{L})$ on each sample were photographed horizontally, and the contact angles were measured. Measurements were performed 6 times on each sample at a temperature of $24^{\circ} \mathrm{C}$ and humidity of $54 \%$.

\section{B. Cell culture}

Ca9-22 cells (RCB1976, RIKEN Cell Bank, Tsukuba, Japan) were cultured in Dulbecco's modified eagle's medium (DMEM; Sigma, St. Louis, MO, USA) containing 10\% fetal bovine serum (CELLect Gold from USA, MP Biomedicals Inc., Solon, OH, USA) and 1\% penicillinstreptomycin-amphotericin B suspension (Wako Pure Chemical Industries, Ltd., Osaka, Japan). The cells were cultured at $37^{\circ} \mathrm{C}$ in $5 \% \mathrm{CO}_{2}$ and $95 \%$ air. Cell growth was assessed daily, and the medium was replaced twice a week. When the cells were $70 \%$ confluent, they were collected using a cell detachment reagent Accumax $^{\mathrm{TM}}$, Funakoshi Co., Ltd., Tokyo, Japan) and cell counting analysis was performed using a hemocytometer. These cells were used in subsequent analyses.

\section{Cell adhesion test}

To measure the change in cell adhesion time on the pattern Ti sheet, we performed a cell adhesion test. First, we added DMEM containing $10 \%$ fetal bovine serum in $1 \%$ penicillin-streptomycin-amphotericin B suspension medium solution to a dish with a fixed $\mathrm{Ti}$ sheet pattern. Ca9-22 cells were added at a density of 5000 cells $/ \mathrm{cm}^{2}$ and cultured at $37^{\circ} \mathrm{C}$ in $5 \% \mathrm{CO}_{2}$ for 1 or $24 \mathrm{~h}$. After cultivation, the cells were fixed with $2.5 \%$ glutaraldehyde solution and stained using a Giemsa stain solution. Adherent cells were observed under an optical microscope (ECLIPSE E200, Nikon, Tokyo, Japan) equipped with a digital camera. We counted the number of adherent cells after 1 or $24 \mathrm{~h}$ of culture using the ImageJ software (NIH, Bethesda, MD, USA) for images shooting the plane, groove, and pillar patterns $\left(5 \times 5 \mathrm{~mm}^{2} /\right.$ field $)$. Error bars indicate the standard deviation of six independent experiments.

\section{Cell morphology}

For SEM analysis of the cells on the pattern Ti sheet, we washed non-adherent cells in PBS after $1 \mathrm{~h}$ of culture and fixed the cells using $2.5 \%$ glutaraldehyde solution. Next, we performed stepped desiccation of a sample every $5 \mathrm{~min}$ at $50 \%, 60 \%, 70 \%, 80 \%, 90 \%, 95 \%$, and $100 \%$, followed by $\mathrm{CO}_{2}$ critical point drying. We performed Pt$\mathrm{Pd}$ sputtering of the sample, and observed the cells by SEM.

\section{E. Immunofluorescence cell staining}

Immunofluorescence staining of the cells was carried out as previously described [12]/ The morphology and vinculin expression of Ca9-22 cells after being seeded for $24 \mathrm{~h}$ on the samples were observed using a fluorescence microscope. The cells were washed twice in PBS and fixed for $10 \mathrm{~min}$ in $3.7 \%$ formaldehyde in PBS. Next, the cells were permeabilized in $0.1 \%$ Triton X-100 (Sigma) in PBS for $5 \mathrm{~min}$ and washed three times in PBS. The cells were then blocked in PBS containing $5 \%$ bovine serum 


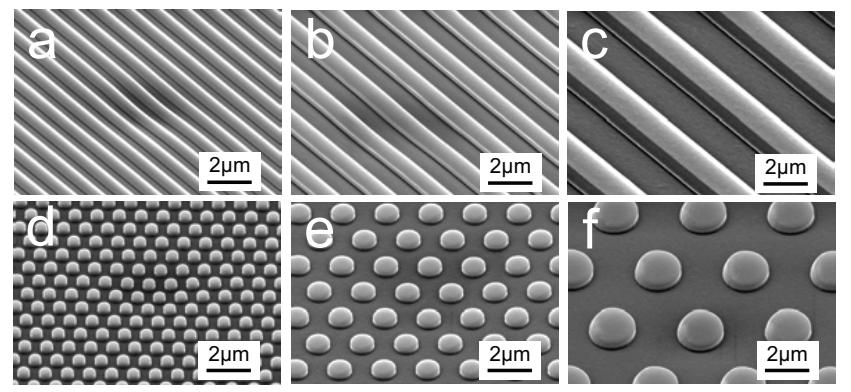

FIG. 2. SEM micrograph of the surface patterns. (a) $500 \mathrm{~nm}$ groove, (b) $1 \mu \mathrm{m}$ groove, (c) $2 \mu \mathrm{m}$ groove, and (d) $500 \mathrm{~nm}$ pillar, (e) $1 \mu \mathrm{m}$ pillar, (f) $2 \mu \mathrm{m}$ pillar. Scale bar: $2 \mu \mathrm{m}$.

albumin for $30 \mathrm{~min}$ and washed once in PBS. Subsequently, the cells were fluorescently stained for detection of vinculin, F-actin, and nuclei at $37^{\circ} \mathrm{C}$ for 60 min using anti-vinculin AlexaFluor 488 (Bioscience, USA), Actistain 555 Fluorescent Phalloidin (Cytoskeleton, Inc., Denver, CO, USA), and DAPI solution (Dojindo, Kumamoto, Japan), respectively, followed by incubation overnight at $4^{\circ} \mathrm{C}$. The samples were observed using a fluorescence microscope (BZ-9000, Keyence Japan). The number of vinculin-positive focal adhesions per cell was estimated on an immunofluorescence cell staining micrograph using ImageJ software. Error bars indicate the standard deviation of $\mathrm{n}=6$.

\section{F. Statistical analysis}

Statistical analysis was performed using GraphPad Prism version 6.05 (GraphPad Software, Inc., La Jolla, CA, USA). All data are presented as the mean and standard deviation. Statistical differences were assessed by one-way ANOVA and Tukey's multiple comparison posthoc test. A value of $p<0.05$ was considered statistically significant.

\section{RESULTS}

\section{A. Fabrication of titanium-coated micro-/nano-patterned sheets}

The patterned Ti-sheets were prepared at the micro/nano-level by nano-imprinting as illustrated in Fig. 1. Patterns on the Ti-sheet were easily molded according to the corresponding patterns of the replica PC mold during the replication process (Fig. 1(a), (b) and (c)). After coating with titanium sputtering (Fig. 1(c)), the patterned Ti-sheet was adhered onto a culture dish and was stable during cell culture.

Surface SEM images of the resulting patterns after coating with titanium sputtering are shown in Fig. 2. Finely grooved and pillared structures were molded from the corresponding shape; the surface of each pattern was smooth at the nano-level. Patterns with widths of $500 \mathrm{~nm}, 1 \mu \mathrm{m}$, and $2 \mu \mathrm{m}$ roughly maintained their ridges and grooves (Fig. 2(a), (b), and (c)). In addition, patterns with widths
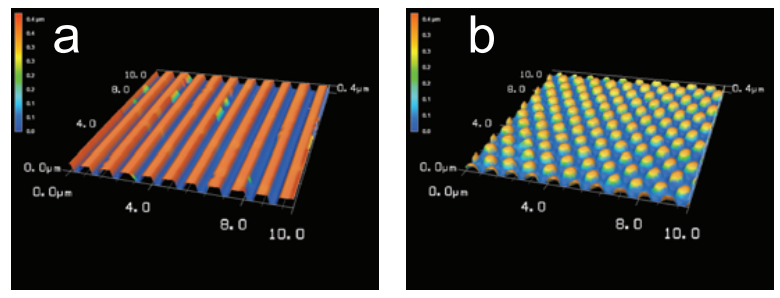

(c)

\begin{tabular}{lllll} 
& & $\mathbf{R}_{\mathbf{a}}(\boldsymbol{\mu} \mathbf{m})$ & \multicolumn{3}{c}{ Height $(\boldsymbol{\mu} \mathbf{m})$} & Width $(\boldsymbol{\mu} \mathbf{m})$ \\
\hline Groove & $500 \mathrm{~nm}$ & $0.18 \pm 0.0052$ & 0.37 & 0.51 \\
\hline Pillar & $500 \mathrm{~nm}$ & $0.12 \pm 0.0066$ & 0.37 & 0.47 \\
\hline
\end{tabular}

FIG. 3. Laser microscope images and analysis of patterned Tisheets. (a) $500 \mathrm{~nm}$ grooved Ti-sheet, (b) $500 \mathrm{~nm}$ pillared Tisheet, (c) surface roughness $\left(R_{a}\right)$, height and width of patterns.

A
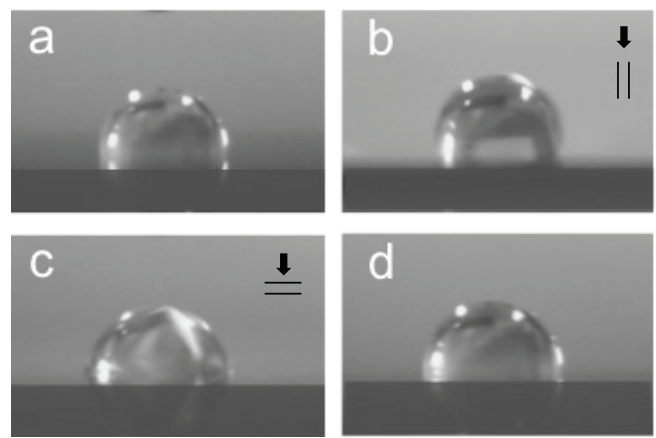

B

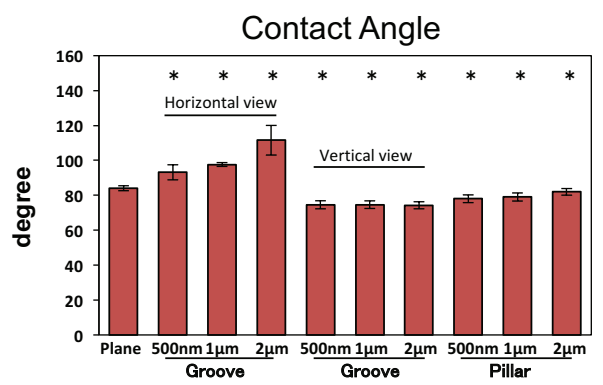

FIG. 4. Contact angle images. (a) Plane Ti-sheet. (b) $500 \mathrm{~nm}$ grooved Ti-sheet (horizontal view for groove). (c) $500 \mathrm{~nm}$ grooved Ti-sheet (vertical view for groove). (d) $500 \mathrm{~nm}$ pillared Ti-sheet. (B) Bar graph summarizing the contact angle data. The contact angle was normalized to that of the plane titanium-coated sheet (control). (* indicates $p<0.05)$

of $500 \mathrm{~nm}, 1 \mu \mathrm{m}$, and $2 \mu \mathrm{m}$ roughly maintained their pillars (Fig. 2(d), (e), and (f)).

Figure 3 shows the laser microscope image and analysis of representative grooved and pillared $\mathrm{Ti}$ sheets with $500 \mathrm{~nm}$ widths. $\mathrm{R}_{\mathrm{a}}$ value of $500 \mathrm{~nm}$ grooves was $0.18 \pm 0.0052 \mu \mathrm{m} ; 500 \mathrm{~nm}$ grooved Ti-sheet had $0.37 \mu \mathrm{m}$ heights and $0.51 \mu \mathrm{m}$ widths. $\mathrm{R}_{\mathrm{a}}$ value of $500 \mathrm{~nm}$ pillars was $0.12 \pm 0.0066 \mu \mathrm{m} ; 500 \mathrm{~nm}$ pillared Ti-sheet had $0.37 \mu \mathrm{m}$ heights and $0.47 \mu \mathrm{m}$ widths. Heights of the grooves and pillars were reduced by approximately $74 \%$ compared to those of the master mold.

Figure 4 shows the contact angle images of patterned Ti-sheets and contact angle data. The contact angle for grooved Ti-sheets (horizontal view for groove) was signif- 
(a)

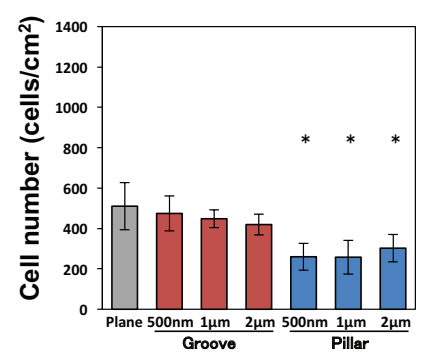

(b)

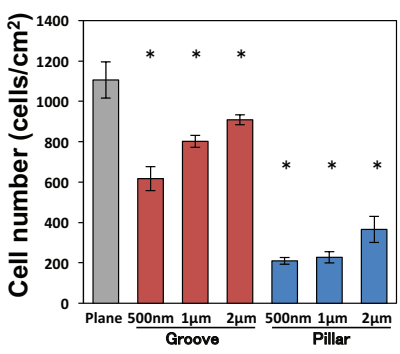

FIG. 5. Number of attached Ca9-22 cells on patterned titanium-coated sheets. (a) After $1 \mathrm{~h}$ incubation (b) after $24 \mathrm{~h}$ incubation. The attached cell number was normalized to that of the plane titanium-coated sheet (control). (* indicates $p<$ $0.05)$.

icantly more hydrophobic than the plane Ti-sheet. The contact angle for $2 \mu \mathrm{m}$ grooved Ti-sheets was the highest of each of the patterned Ti-sheets. However, the contact angle for grooved Ti-sheets (vertical view for groove) was more hydrophilic than the plane Ti-sheet. The contact angle for the pillared Ti-sheet was significantly more hydrophilic than the plane Ti-sheet. However, the contact angle for pillared Ti-sheet was not significantly different among the $500 \mathrm{~nm}-, 1 \mu \mathrm{m}-$, and $2 \mu \mathrm{m}$-pillared patterns.

\section{B. Cell adhesion and proliferation test}

Figure 5 shows the cell adhesion count in Ca9-22 cells after 1 and $24 \mathrm{~h}$, on the patterned Ti sheet. After $1 \mathrm{~h}$ of culture, no significant differences in the cell adhesion count on the $500 \mathrm{~nm}, 1 \mu \mathrm{m}$, and $2 \mu \mathrm{m}$ grooved Ti sheet were observed compared with the plane Ti-sheets (control) $(p>0.05)$. However, after $24 \mathrm{~h}$, the cell adhesion count on each groove was lower than that of the plane $(p<0.05)$. In addition, the cell adhesion count on the groove after $24 \mathrm{~h}$ showed a lower cell adhesion count than on the smaller groove width.

After 1 and $24 \mathrm{~h}$ of culturing, the cell adhesion count on the $500 \mathrm{~nm}, 1 \mu \mathrm{m}$, and $2 \mu \mathrm{m}$ pillared Ti sheet was significantly less than that on the plane Ti-sheets (control) $(p<0.05)$. After $24 \mathrm{~h}$ of culture, the $2-\mu \mathrm{m}$ pillared cell adhesion count was highest at $500 \mathrm{~nm}$ and $1 \mu \mathrm{m}$. While the plane and grooved cell adhesion count increased by nearly double from 1 to $24 \mathrm{~h}$, the cell adhesion count on the pillar showed no increase during the same time.

\section{SEM image of Ca9-22 cells}

Figure 6 shows SEM images of Ca9-22 cells after $1 \mathrm{~h}$ of culturing on the Ti sheet. In the plane after $1 \mathrm{~h}$, the cells assumed a surface morphology on the dome that lifted the central part of the cell, and filopodia extending in all four directions were observed (Fig. 6(a)). In grooves with $500 \mathrm{~nm}, 1 \mu \mathrm{m}$, and $2 \mu \mathrm{m}$ widths, the cells assumed an elliptical shape in a parallel direction to the groove and extended along the groove. No major differences in cell size related to groove size were observed (Figs. 6(b), (c), and
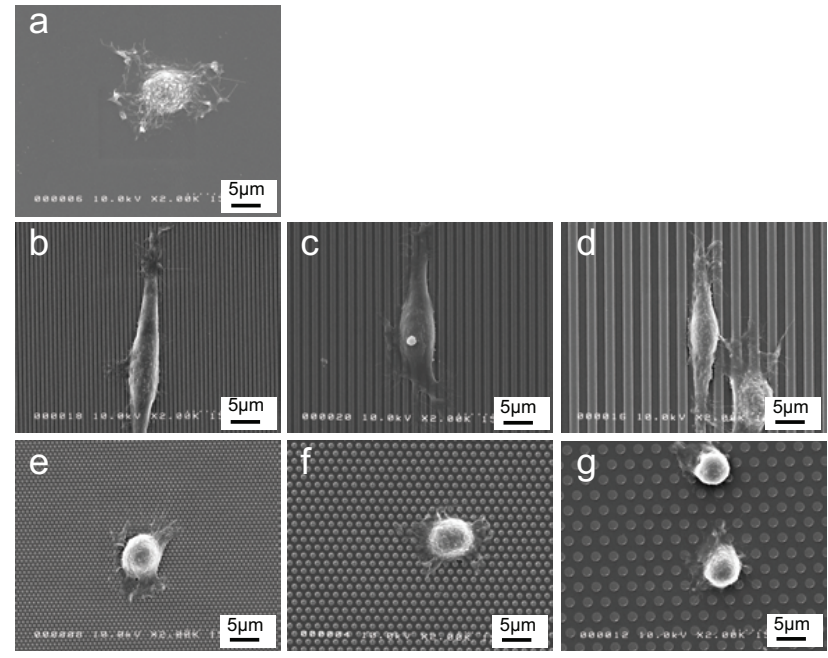

FIG. 6. SEM images of cell morphology of the patterned Tisheets after $1 \mathrm{~h}$ incubation. (a) Plane Ti-sheet, (b) $500 \mathrm{~nm}$ grooved Ti-sheet, (c) $1 \mu \mathrm{m}$ grooved Ti-sheet, (d) $2 \mu \mathrm{m}$ grooved Ti-sheet, (e) $500 \mathrm{~nm}$ pillared Ti-sheet, (f) $1 \mu \mathrm{m}$ pillared Tisheet, (g) $2 \mu \mathrm{m}$ pillared Ti-sheet.

(d)). In the $500 \mathrm{~nm}, 1 \mu \mathrm{m}$, and $2 \mu \mathrm{m}$ wide pillar shapes, the cells assumed a dome-shaped surface morphology and were relatively small compared to the plane-shaped cells. Moreover, the filopodia were also reduced compared to the cells on the plane (Figs. 6(e), (f), and (g)).

\section{Focal adhesion by immunofluorescence cell staining}

Figure 7 shows the immunofluorescence cell staining micrographs of Ca9-22 cells on patterned Ti-sheets after incubation for $24 \mathrm{~h}$. Cells expressed a large number of focal adhesion protein. Vinculin, F-actin, and nuclei were distinctly stained. Ca9-22 cells on the plane Ti-sheet were large and extended (Fig. 7(a)). Cells on $500 \mathrm{~nm}$-grooved Ti-sheet were large and elongated with the vinculin spot and $\mathrm{F}$-actin extending in a direction parallel to the grooves (Fig. 7(b)). Cells on $500 \mathrm{~nm}$ pillared Ti-sheet showed a morphology similar to that of cells on the plane Ti-sheets (Fig. 7(c)). However, the number of vinculin spots that extended on the pillared Ti-sheet was lower than that on the plane Ti-sheet. Figure 8 shows the number of vinculin-positive focal adhesions per cell on the patterned Ti sheets after incubation for $24 \mathrm{~h}$. The numbers of vinculin-positive focal adhesions on the grooved $\mathrm{Ti}$ sheets and pillared $\mathrm{Ti}$ sheets were lower than that on the plane $\mathrm{Ti}$ sheet $(p<0.05)$. The number of vinculinpositive focal adhesions on the plane $\mathrm{Ti}$ sheet was the highest in the patterns.

\section{DISCUSSION}

In this study, we fabricated $\mathrm{Ti}$ sheets with grooved and pillared structures by nanoimprinting. However, the heights of the grooves and pillars were reduced compared to those of the master mold (Fig. 3). In our method, it 

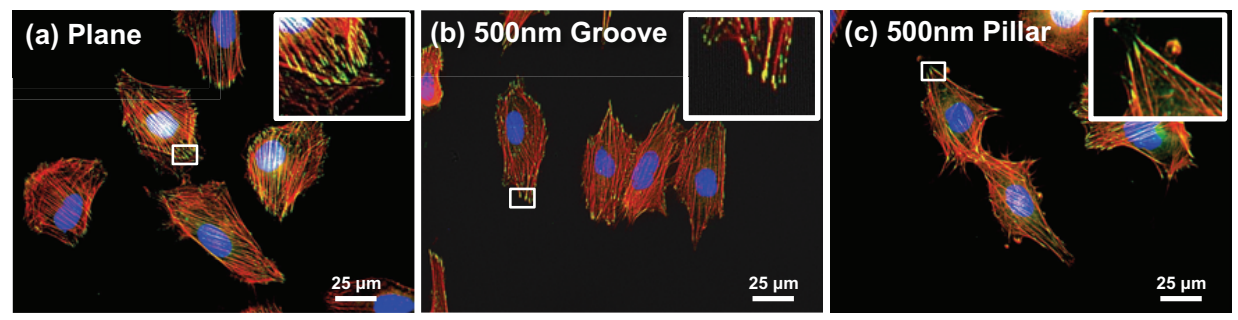

FIG. 7. Immunofluorescence micrograph of Ca9-22 cells on Ti-sheets after $24 \mathrm{~h}$ incubation. (a) Plane Ti-sheet, (b) $500 \mathrm{~nm}$ grooved Ti-sheet, (c) $500 \mathrm{~nm}$ pillared Ti-sheet. Green: vinculin enclosed in a square, red: F-actin, blue: nuclei. Scale bars: $25 \mu \mathrm{m}$.

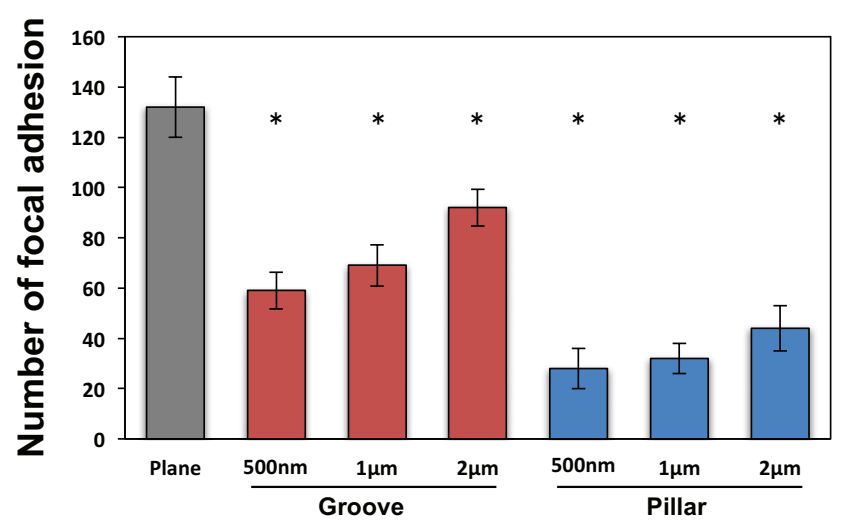

FIG. 8. Number of vinculin-positive focal adhesions in Ca922 cells on Ti-sheets. The number of vinculin-positive focal adhesions was lower on grooved and pillared Ti-sheets than on plane Ti-sheets $(p<0.05)$. The number of focal adhesions was normalized to that of the plane Ti-sheet (control) $(*$ indicates $p<0.05)$.

was difficult to prepare patterns of smaller sizes. This method should be improved in future studies.

This study showed that the contact angles for grooved Ti-sheets (vertical view for groove) and pillared Ti-sheet were significantly more hydrophilic than the plane Tisheet (Fig. 4). Interestingly, in the grooved Ti-sheet, the contact angle for grooved Ti-sheets (vertical view for groove) was more hydrophilic than for grooved Ti-sheets (horizontal view for groove). Thus, groove structures appeared to affect cell alignment along the groove direction (Figs. 6(b), (c), and (d)).

SEM analysis of the surface morphology taken on the dome on the plane showed that the cell surface of cultured cells was rough, with filopodia around the entire plasma membrane (Fig. 6(a)). This is in agreement with the epithelial-like cell findings reported previously [7]. In the grooved substrate shape, it was observed that the cells expanded and oriented along the groove (Fig. 6(b), (c), and (d)). Similarly to osteoblast $[5,13]$ or fibroblast cells $[6,14]$, which are frequently used in research studies, an orientation on the groove substrate is suggested, even in epithelial cells. In addition, as in a study showing that epithelial cell adhesion and proliferation was suppressed on rough surfaces, it was observed that cells on the pillar were smaller than those cultured on the plane, and that there were few filopodia (Figs. 6(e), (f), and (g)).

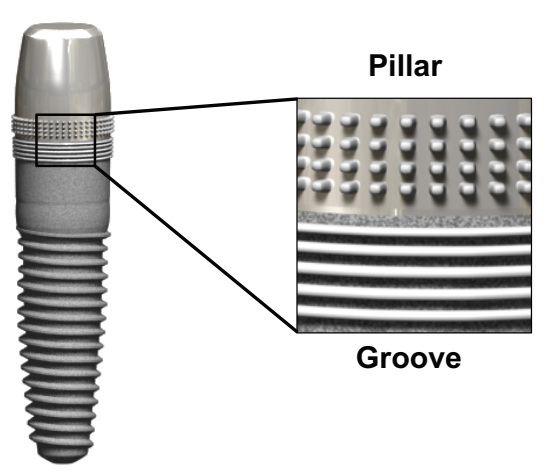

FIG. 9. Illustration for surface structures of dental implant body and abutment.

Thus, the pillared structure had rough surfaces in epithelial cells, indicating inhibition of adhesion and proliferation.

Grooved structures for the implant body surface suppress epithelial deep proliferation, arrays in the fiber perpendicular direction, and travel of osteoblast cells to promote morphological formation towards the deep parts of the grooves.

Because the surface structure of the area is adjacent to bone, we aimed to produce a structure with roughly structured surfaces other than grooves and pillars [15], promoting in every case bone formation by the microenvironment and maintaining an interlocking force between the bone and implant body [5-11].

No comparative studies have investigated the cell adhesion and dynamics of epithelial cells on titanium surfaces.

In this study, we attached a nano-pattern structure to a titanium surface and showed that it affected the adhesion of Ca9-22 epithelial cells. Previous studies using osteoblast and fibroblast cells demonstrated that attachment of grooved and pillared structures increased the cell adhesion count to a greater extent than smooth titanium $[6,16]$. In this study, while the epithelial cells at $1 \mathrm{~h}$ after culture did not show a significant difference in cell adhesion count on the groove compared with the plane, the cell adhesion count on the pillar was smaller than that on the plane. After $24 \mathrm{~h}$ of culture, the cell adhesion count on the grooved and pillar was significantly lower than that compared to on the plane. On the pillar, no increase in cell adhesion count was observed at 1 or 24 h (Fig. 5). 
Vinculin is a major structural component of focal contacts with various binding sites for intracellular molecules and is a type of connecting molecule [4]. The present study showed that the number of vinculin-positive focal adhesions on the plane Ti-sheets was higher than that on the patterned Ti sheets (Fig. 7 and 8). A previous study observed vinculin-positive focal adhesions of human bone cells [17]. Interestingly, a similar trend was observed for cell adhesion count and the number of vinculin-positive focal adhesions after incubation for $24 \mathrm{~h}$. These results indicate that focal adhesion formation is involved in cell adhesion.

These results suggest that in epithelial cell adhesion and proliferation, the flat plane surface is better than the titanium surface with a grooved and pillared structure. One study reported that the use of a grooved structure in the implant abutment surface structure suppressed epithelial downgrowth [18]. This indicates that use of a pillared structure in the implant abutment surface structure can suppress epithelial deep proliferation.

However, the suitable size for the grooves and pillars in cell adhesion and proliferation has not been determined. In this study, we only analyzed groove widths of $500 \mathrm{~nm}$, $1 \mu \mathrm{m}$, and $2 \mu \mathrm{m}$. In addition, attachment of microstructures to the surface led to bacterial adhesion. Previous studies showed that the size of surface bumps and indentations are related, with the indentation width easy to attach at approximately $3 \mu \mathrm{m}$, and bacteria are unable to adhere to nano-structures of $0.4 \mu \mathrm{m}$ or less [19].

Further studies are needed to evaluate the production of patterns smaller than $500 \mathrm{~nm}$ and assess the performance of biomechanics tests or in vivo experiments on microand nano-grooves and pillars.

\section{CONCLUSION}

In this study, we found that the cell adhesion count on the $500 \mathrm{~nm}, 1 \mu \mathrm{m}$, and $2 \mu \mathrm{m}$ width grooved and pillared Ti sheet was reduced compared to cells cultured on plane Ti sheets. Furthermore, Ca9-22 cells showed a decreased cell adhesion count and focal adhesions on the grooves and pillars. Our results provide further insight into how structuring of implant interfaces at the micro-/nano-scale can prevent soft tissue encapsulation which limits the lifetime of many implants. These results suggest that grooved and pillared structures on the titanium surface increases the suppression of epithelial deep cell proliferation. In the future, we will develop the appropriate surface structures of dental implant body and abutment for prevention of peri-implantitis (Fig. 9).

\section{ACKNOWLEDGMENTS}

This study was funded by JSPS KAKENHI Grant No. 26670829, No. 16H05518, and No. 25463047. The analysis of surface profiles for different patterns was carried out with a laser microscope at the OPEN FACILITY, Hokkaido University Sousei Hall.
[1] A. D. Pye, D. E. A. Lockhart, M. P. Dawson, C. A. Murray, and A. J. Smith, J. Hosp. Infect. 72, 104 (2009).

[2] Y. Shibata and Y. Tanimoto, J. Prosthodont. Res. 59, 20 (2015).

[3] L. Larsson, A. M. Decker, L. Nibali, S. P. Pilipchuk, T. Berglundh, and W. V. Giannobile, J. Dent. Res. 95, 255 (2016).

[4] T. Goto, M. Yoshinari, S. Kobayashi, and T. Tanaka, Biomed. Mater. Eng. 14, 537 (2004).

[5] K. Matsuzaka, M. Yoshinari, M. Shimono, and T. Inoue, J. Biomed. Mater. Res. A. 68, 227 (2004).

[6] M. Yoshinari, K. Matsuzaka, T. Inoue, Y. Oda, and M. Shimono, J. Biomed. Mater. Res. A. 65, 359 (2003).

[7] B. Baharloo, M. Textor, and D. M. Brunette, J. Biomed. Mater. Res. A. 74, 12 (2005).

[8] B. Chehroudi, T. R. L. Gould, and D. M. Brunette, J. Biomed. Mater. Res. 25, 387 (1991).

[9] B. D. Boyan, L. F. Bonewald, E. P. Paschalis, C. H. Lohmann, J. Rosser, D. L. Cochran, D. D. Dean, Z. Schwartz, and A. L. Boskey, Calcif. Tissue. Int. 71, 519 (2002).

[10] F. P. Nothdurft, D. Fontana, S. Ruppenthal, A. May, C. Aktas, Y. Mehraein, P. Lipp, and L. Kaestner, Clin. Im- plant. Dent. Relat. Res. 17, 1237 (2015).

[11] A. Furuhashi, Y. Ayukawa, I. Atsuta, H. Okawachi, and K. Koyano, Odontology 100, 199 (2012).

[12] N. Kaga, T. Akasaka, R. Horiuchi, Y. Yoshida, and A. Yokoyama, Nano Biomedicine 8, 74 (2016).

[13] X. Lu and Y. Leng, J. Biomed. Mater. Res. A. 66, 677 (2003).

[14] E. Kokubu, D. W. Hamilton, T. Inoue, and D. M. Brunette, J. Biomed. Mater. Res. A. 91, 663 (2009).

[15] N. Lumbikanonda and R. Sammons, Int. J. Oral Maxillofac. Implants. 16, 627 (2001).

[16] J. Chen, J. P. Ulerich, E. Abelev, A. Fasasi, C. B. Arnold, and W. O. Soboyejo, Mater. Sci. Eng. C. 29, 1442 (2009).

[17] K. Anselme, M. Bigerelle, B. Noel, E. Dufresne, D. Judas, A. Iost, and P. Hardouin, J. Biomed. Mater. Res. 49, 155 (2000).

[18] M. Nevins, M. L. Nevins, M. Camelo, J. L. Boyesen, and D. M. Kim, Int. J. Periodontics Restorative Dent. 28, 111 (2008).

[19] M. Lorenzetti, I. Dogša, T. Stošicki, D. Stopar, M. Kalin, S. Kobe, and S. Novak, ACS Appl. Mater. Interfaces. 7, 1644 (2015). 